This is the Pre-Published Version

\title{
VQ-RED: An Efficient Virtual Queue Management Approach to Improve Fairness in Infrastructure WLAN
}

\author{
Xiaoyang Lin, Xiaolin Chang, and Jogesh K. Muppala \\ Dept. of Computer Science \\ Hong Kong University of Science and Technology \\ Clear Water Bay, Kowloon, Hong Kong \\ Email: \{xylin,changxl,muppala\}@cs.ust.hk
}

\begin{abstract}
In this paper, we consider two fairness problems (downlink/uplink fairness and fairness among flows in the same direction) that arise in the infrastructure $W L A N$. We propose a virtual queue management approach, named VQ-RED to address the fairness problems. We demonstrate the effectiveness of our approach by conducting a series of simulations. The results show that compared with standard $D C F, V Q-$ RED not only greatly improves the fairness, but also reduces packet delays.
\end{abstract}

\section{Introduction}

Wireless local area networks (WLANs) based on the IEEE 802.11 standard [1] are experiencing widespread deployment thanks to the ease-to-use of the technology, the low cost of the equipment and the real need for such services. Unlicensed spectrum and the interoperability granted by adherence to standards and to certifications (such as Wi-Fi) also contributed to this growth. Currently the focus is turning to deploy these networks in "hot spots" such as airports, hotels, cafes, libraries and other areas which provides the nomadic users with public access to the Internet. This "hot spots" coverage is based on the Access Point devices which provide the mobile nodes with access to the wired network. This scenario is often called infrastructure WLAN which is different from ad hoc WLAN in which the mobile nodes communicate directly with each other, without the help of an Access Point.

The IEEE 802.11 standard provides two coordination functions for medium access: distributed coordination function (DCF) and point coordination function (PCF). In DCF a set of wireless stations communicate with each other using a contention-based channel-access method, namely carrier sense multiple access with collision avoidance (CSMA/CA). The CSMA/CA guarantees that all the stations in WLAN get equal opportunity to access the medium. PCF is a contention free access method designed for infrastructure WLANs. It gives more control to the Access Point which takes the role of a scheduler. In PCF, the Access Point can determine which station can access the medium at what time by means of a polling technique.

In the infrastructure WLANs, the Access Point plays the important role for relaying the traffic between the mobile nodes (wireless stations) and the wired network which results in asymmetric traffic load between the Access Point and single mobile node. Although PCF is designed for infrastructure networks, the problem is that currently most of the WLAN cards do not support the PCF mode. With DCF mode, the CSMA/CA mechanism makes the Access Point and mobile nodes have the same priority to access the medium. This leads to the significant unfair WLAN bandwidth distribution between uplink and downlink flows. Downlink (resp. uplink) flows represents those flows whose sources are wired (resp. mobile) nodes and receivers are mobile (resp. wired) nodes; downlink (resp. uplink) packets represents packets sent from wired (resp. mobile) nodes to mobile (resp. wired) nodes. We refer to the fair WLAN bandwidth distribution between downlink and uplink flows as downlink/uplink fairness. Consider the situation when there are $\mathrm{n}$ mobile senders and $\mathrm{n}$ mobile receivers. In this case, the Access Point and the $\mathrm{n}$ mobile senders share the bandwidth equally, namely, each gets $1 /(n+1)$ of the total available WLAN bandwidth. And the $1 / n+1$ of the total bandwidth possessed by the Access Point is shared by the $\mathrm{n}$ mobile receivers. The bandwidth ratio between the uplink flows and downlink flows is n, which is very unfair since the numbers of uplink flows and downlink flows are identical. 
The downlink/uplink fairness problem is compounded further in the case of TCP flows because of the greedy closed loop control nature of TCP [2]. The available bandwidth of 802.11 WLAN is much smaller than that of wired networks. There are three reasons for this: (1) ideally the maximum bandwidth of IEEE $802.11 \mathrm{~b}$ which is the most deployed WLAN standard can achieve $11 \mathrm{Mbps}$, which is relatively small compared to the widely-deployed $100 \mathrm{Mpbs}$ Fast Ethernet in a corporate networks; (2) the WLAN bandwidth is shared among the uplink and downlink flows within a wireless cell (half-duplex); (3) the acknowledge overhead of CSMA/CA mechanism consumes additional bandwidth, compared to CSMA/CD mechanism. This bandwidth disparity between wired networks and WLAN makes the Access Point becomes the potential bottleneck in the downlink direction. The downlink TCP packets and the TCP ACK packets for uplink flows queue in the buffer of Access Point. Therefore the buffer size of the Access Point plays an important role for the TCP downlink/uplink fairness [2].

Besides the downlink/uplink fairness, another fairness problem in the infrastructure WLAN is the fairness among flows in the same direction (uplink or downlink). When the MAC level is operating in congestion situation, the TCP is unable to provide a fair share of bandwidth among competing flows: some flows may grab most of the bandwidth while other flows may not be able to transmit for a long time [3].

In this paper, we propose a software upgrade-based approach, named VQ-RED to address the fairness issues in IEEE 802.11 infrastructure WLAN with DCF access. Our approach is only deployed on AP while we do not make any change to the MNs.

The rest of the paper is organized as follows. We review the related work in Section 2. In Section 3, we propose VQ-RED. Simulation results are given in section 4. Section 5 is the conclusion to our paper.

\section{Related Work}

In the past several years, extensive studies have been carried out to study the fairness issues in cellular network, infrastructure WLANs and ad hoc WLANs [4]. This section focuses on the research related to infrastructure WLANs. Scheduling mechanisms aimed at providing fair channel access have been proposed at the LL and MAC layers (see the references cited in [4]). They focus on the fairness among the mobiles senders in a WLAN.

Some authors (see, for example, [5] and the references cited therein) have observed downlink congestion caused by the speed mismatch between a wired network and WLAN at the AP. They explored applying active queue management (AQM) to control congestion and achieve some quality of service $(\mathrm{QoS})$, such as low delay, low packet loss rate and high goodput. They only consider the network scenarios of pure downlink flows.

Meanwhile, several research efforts have been made to alleviate downlink/uplink unfairness. The authors in [4] [6] [7] proposed different methods at the MAC layer. The method in [7] is based on the PCF mode. The methods in [4] and [6] are based on the DCF mode but only paid attention to the benefit of downlink flows. In the WLAN for TCP flows where the downlink congestion is effectively controlled, downlink flows may obtain more WLAN bandwidth than uplink flows in some scenarios even when the number of flows in two directions is identical. In addition, the method in [4] required the cooperation among MNs and the AP. The closest works to ours are the ones in [2] and [3], which explored methods above the MAC layer in the WLAN for TCP flows to alleviate the downlink/uplink fairness. The authors in [2] assumed that the AP knows the number of active TCP flows $n$, then they proposed to feedback the information of $\lfloor$ buffer_size/ $n\rfloor$ via ACK packets to the senders in order to alleviate downlink/uplink fairness. The authors in [3] assumed that the knowledge of the maximum achievable WLAN bandwidth is available. Then an IP level rate-limiter is used to limit the rate of uplink flows allowed to be sent out from the AP to the wired network. One problem is the low WLAN bandwidth utilization when the maximum sending rate of all wired senders is less than half of the maximum achievable WLAN bandwidth. To the best of our knowledge, the fairness among flows in the same direction is discussed only in [3].

\section{VQ-RED}

In this section, we present our approach, named VQ-RED to address the fairness issues in infrastructure WLANs. Our approach is deployed on the AP (more accurately, deployed at the gateway of the incoming buffer and outgoing buffer in the AP) and does not require any change to be made to the MNs.

The architecture of VQ-RED is given in Figure 1. VQ-RED consists of three components: Virtual queues (VQs), Classifier and Virtual Queue Manager (VQM). They are explained as follows:

- VQ: The VQ is a data structure to represent a real queue. Actually it is kept track of only by its length. Therefore, there is no real enqueue and dequeue operation for VQ. The "enqueue" (resp. "dequeue") operation is achieved only by 
increasing (resp. decreasing) the queue length. Each virtual queue is for a particular active flow. In this paper, each flow is identified by the pair of the sender and the receiver. A flow may contain TCP traffic, UDP traffic or both.

- Classifier: The Classifier classifies each packet and dispatches it to a proper VQ. If it encounters a packet that belongs to a new flow, it will notify the VQM to create a new VQ for this new flow.

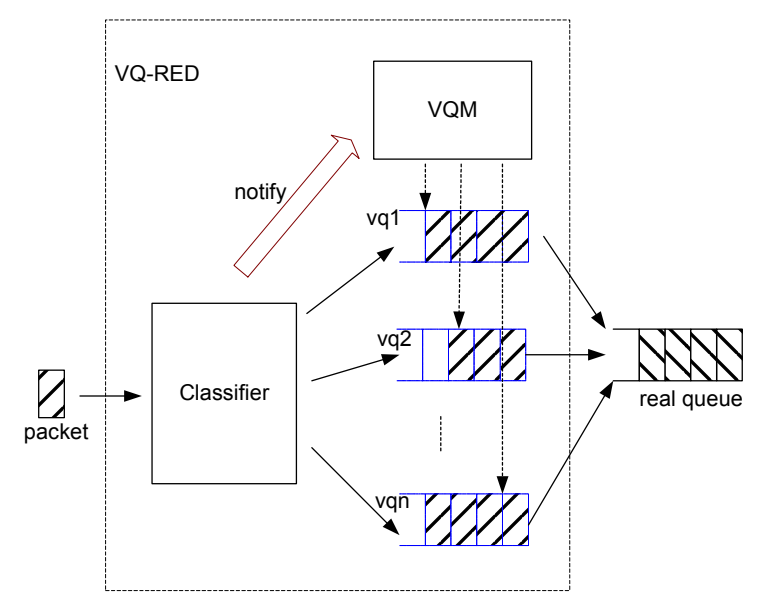

Figure 1: VQ-RED Architecture

- VQM: it is the center of the whole approach. It is responsible for the following tasks:

1. Estimate the average rate: the VQM periodically estimate the average rate of all the flows (including uplink flows and downlink flows) using the following formula:

rate $=$ rate $* 0.1+\frac{\text { sum }}{\text { flowNum } * \text { period }} * 0.9$

Here "rate" represents the average rate of all the flows, "sum" represents the sum of the length of all the arriving packets during this period, "period" represents the length of the period and "flowNum" represents the number of the active flows which is actually the number of VQs.

2. Create VQs: When the VQM receives a notification from the Classifier that there comes a new flow, it will create a new VQ for this connection

3. Delete VQs: The VQM will delete some VQ if this VQ is not longer active. A VQ is assumed to be inactive if it does not "receive" packets for a long time. In order to achieve this, the VQM set a timer for each VQ, when timeout happens, the VQM will delete the corresponding VQ. When a VQ "receives" a packet, its timer is reset.

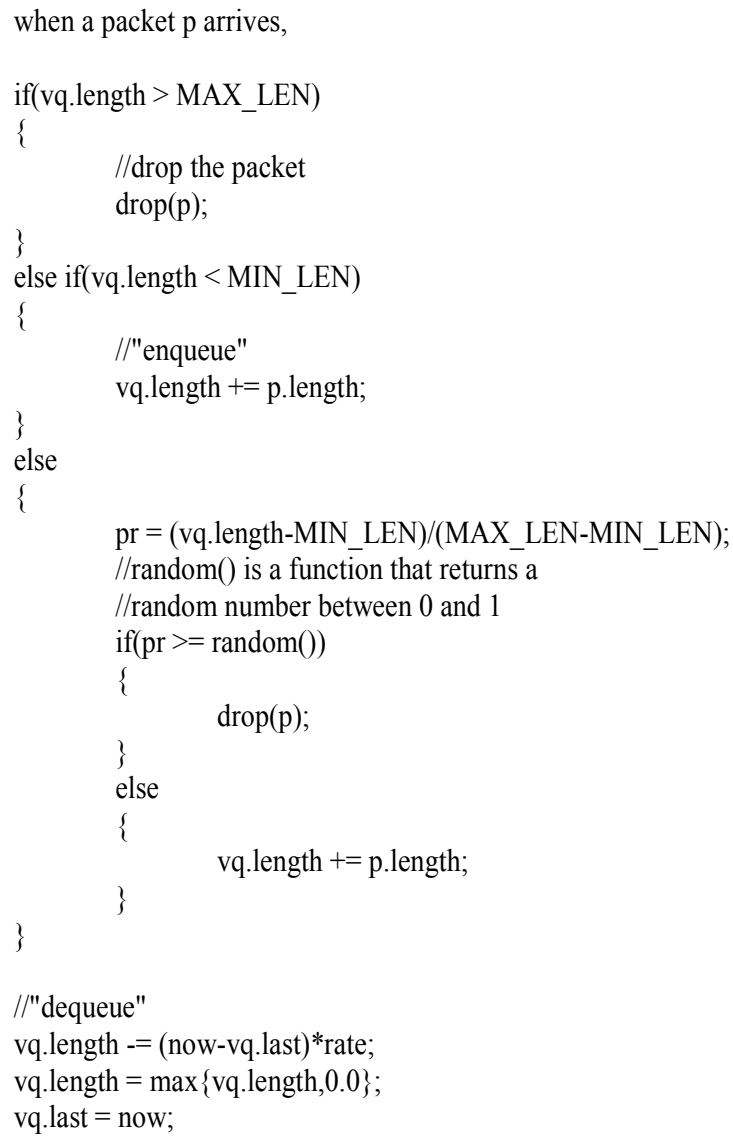

\section{Figure 2 VQM algorithm}

4. Manage VQs: The VQM manages each VQ using the algorithm given in Figure 2. When a packet is dispatched (by the Classifier) to the corresponding $\mathrm{VQ}$, the VQM firstly examines the length of the VQ to determine whether to drop the packet or not. There are three situations: (1) If the length is larger than an upper threshold value MAX_LEN, the packet is dropped directly; (2) If the length is smaller than a lower threshold value MIN_LEN, the packet is "enqueued" to the VQ. (3) Otherwise, the length of VQ is between the MIN_LEN and MAX_LEN and the packet is dropped with a probability that is based on the length. The longer the length is, the higher the dropping probability (Note that in Figure 2, the function "random()" returns a random value between 0 and 1). After the dropping (or "enqueue") operation, the VQM should do the "dequeue" operation which determines how many packets should "leave" the VQ since last time it is accessed. To achieve this, the VQM should keep track of the last access time of each VQ and use the following formula to simulate this operation: vq.length $-=($ now - vq.last $) *$ rate 
here "now" represents the current time, "vq.last" represents the last access time of the vq and "rate" is the average rate of all the flows. This VQ management algorithm is similar with the Random Early Discard (RED). Hence we name our approach VQ-RED.

VQ-RED treats all the competing flows (uplink flows and downlink flows) fairly through managing their corresponding VQs. It punishes the arbitrary flows and gives more benefits to the weak flows. In this way it guarantees the fairness among the flows.

\section{Simulation Results}

In this section, we use ns2 [8] to evaluate VQ-RED. The network topology is given in Figure 3. The wireless bandwidth is set to $11 \mathrm{Mbps}$ while the wired bandwidth is set to $100 \mathrm{Mbps}$. Let $n$ denote the number of wired nodes and let $m$ denote the number of wireless nodes. Each node sends both TCP and UDP traffic. The TCP (resp. UDP) flows from $\mathrm{S}_{\mathrm{A} 1} \ldots \mathrm{S}_{\mathrm{An}}$ to $\mathrm{D}_{\mathrm{A} 1} \ldots$ $\mathrm{D}_{\mathrm{An}}$ are TCP (resp. UDP) downlink flows. The TCP (resp. UDP) flows from $S_{B 1} \ldots S_{B m}$ to $D_{B 1} \ldots D_{B m}$ are TCP (resp. UDP) uplink flows. All TCP flows are FTP flows using TCP-Reno and all UDP flows are CBR flows. IP Packet size is 1500 bytes.
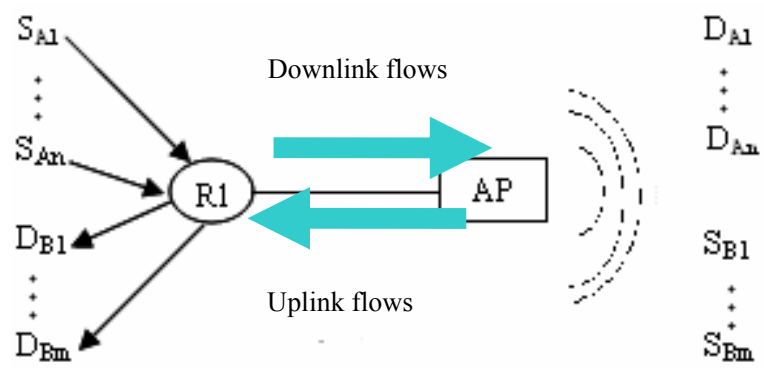

Figure 3 The network topology

We do simulations under VQ-RED and standard DCF respectively and compare their results. In the simulations, we measure the fairness for TCP flows and UDP flows respectively. We measure the TCP (resp. UDP) downlink/uplink fairness by the ratio of the average goodput of TCP (resp. UDP) downlink flows and the average goodput of TCP (reps. UDP) uplink flows. And we use the fairness index [9] to measure the fairness among TCP (resp. UDP) flows in the same direction (uplink or downlink). The closer the result is to 1 , the more fairness is achieved.

\subsection{TCP and UDP Flows}

We set the sending rate of UDP traffic as $100 \mathrm{kbps}$ and let $n=10$ and $m=10$. We do simulations with different buffer sizes at the AP. The results are given from Figure 4 to Figure 11. Figure 4 shows the TCP (resp. UDP) fairness among flows in the same direction. Figure 5 shows the TCP (resp. UDP) downlink/uplink fairness. Figure 6 shows the achieve TCP goodput, UDP goodput and total goodput. Figure 7 shows the average round trip time for TCP flows. Figure 8, Figure 9, Figure 10 and Figure 11 respectively show the goodput distribution among TCP (or UDP) flows under VQ-RED (or standard DCF) when the buffer size is set to 100 .

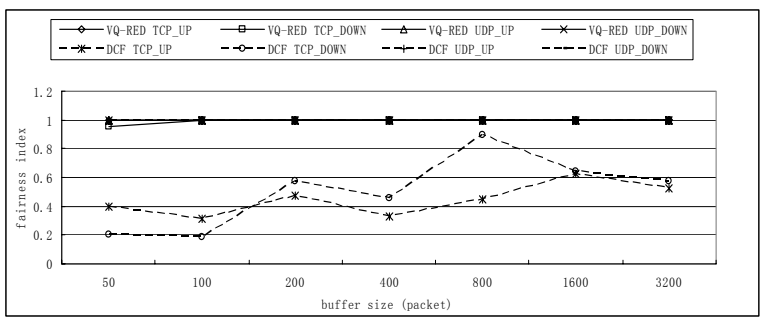

Figure 4 fairness among flows in the same direction $(n: m=10: 10$, sending rate of UDP traffic is $100 \mathrm{kbps}$ )

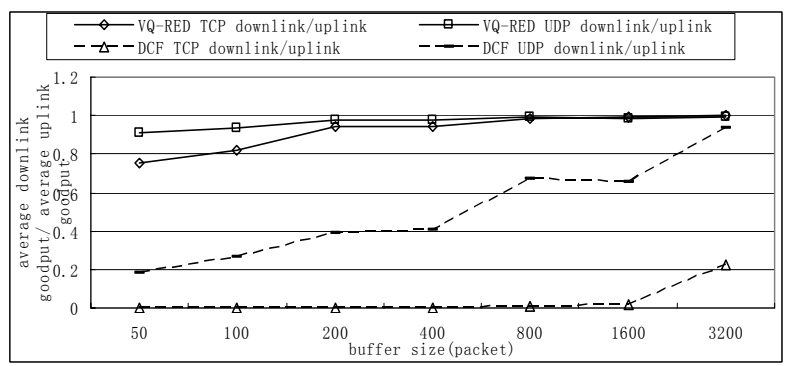

Figure 5 downlink/uplink fairness $(\mathrm{n}: \mathrm{m}=10: 10$, sending rate of UDP traffic is $100 \mathrm{kbps}$ )

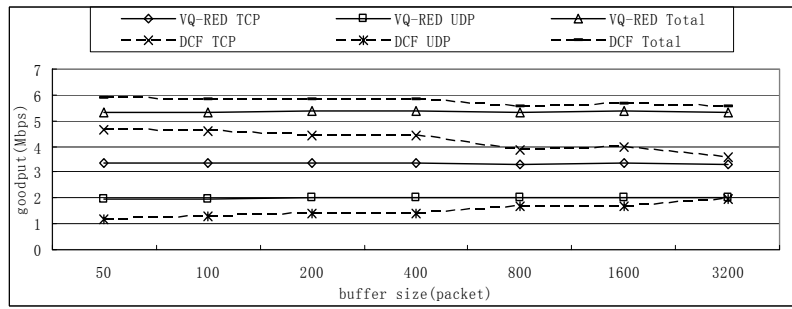

Figure 6 total achieved goodput $(n: m=10: 10$, sending rate of UDP traffic is $100 \mathrm{kbps}$ ) 


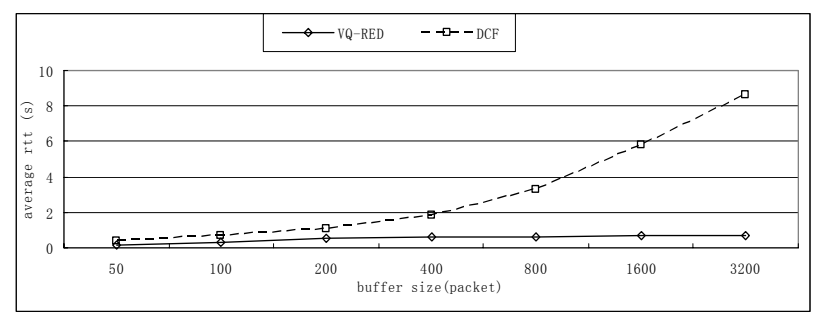

Figure 7 average $r t$ delay $(n: m=10: 10$, sending rate of UDP traffic is $100 \mathrm{kbps}$ )

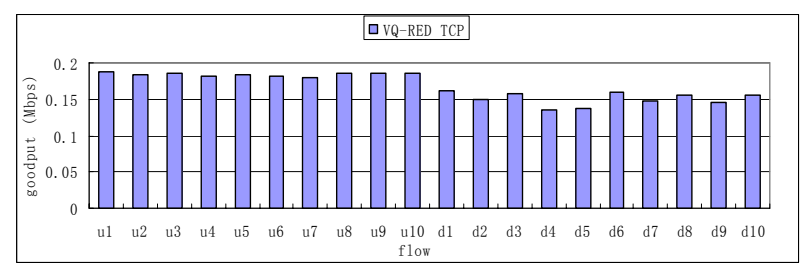

Figure 8 goodput of each TCP flow under VQRED (AP buffer size $=100, \mathrm{n}: \mathrm{m}=10: 10$, UDP sending rate is $100 \mathrm{kbps})$

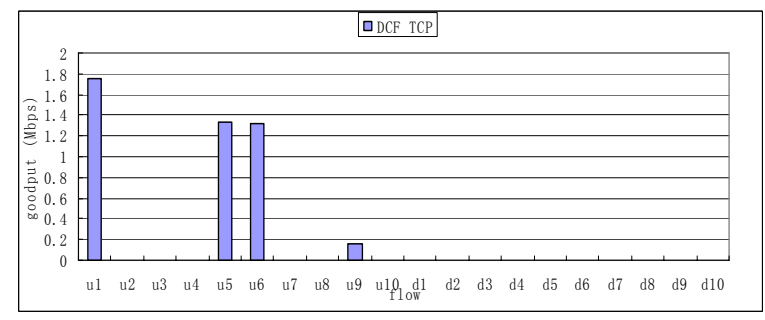

Figure 9 goodput of each TCP flow under DCF (AP buffer size $=100, \mathrm{n}: \mathrm{m}=10: 10$, sending rate of UDP traffic is 100kbps)

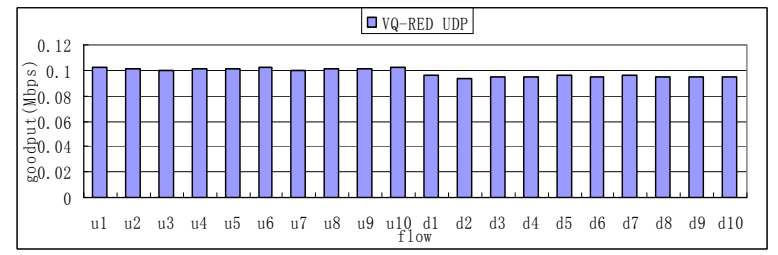

Figure 10 goodput of each UDP flow under VQ-RED (AP buffer size $=100, n: m=10: 10$, sending rate of UDP traffic is $100 \mathrm{kbps}$ )

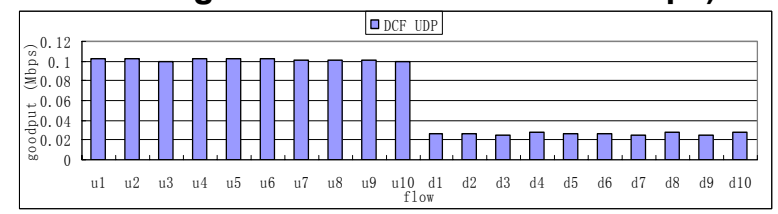

Figure 11 goodput of each UDP flow under DCF (AP buffer size $=100, \mathrm{n}: \mathrm{m}=10: 10$, sending rate of UDP traffic is $100 \mathrm{kbps}$ )
From the simulation results, we reach the following conclusions:

- Fairness among flows in the same direction: for UDP traffic, both VQ-RED and standard DCF can achieve very good fairness in both uplink and downlink directions. However, for TCP traffic, under DCF, the bandwidth is unfairly distributed among the flows in the same direction, some flows grab most of the bandwidth while others get very little (because the TCP downlink flows get too little bandwidth compared with TCP uplink flows, we can not see their distribution from Figure 9). The situation is improved greatly by VQ-RED. We can see that VQ-RED can almost achieve total fairness among TCP flows no matter what size of the buffer is.

- Downlink/uplink fairness: under DCF, the uplink TCP flows totally overwhelm the downlink TCP flows and this situation is improved slightly when the buffer size becomes larger; although the uplink UDP flows can achieve about 100kbps which is the sending rate, the downlink UDP flows are not able to achieve this. Both the downlink/uplink fairness for TCP traffic and UDP traffic can be greatly improved by VQ-RED. With VQ-RED, the average goodput of uplink TCP flows and downlink TCP flows are very close and all the UDP flows (both uplink and downlink) can get to about 100kbps.

- Average delay: we also compare the average round trip time (rtt) delay between VQ-RED and DCF. The result in Figure 7 shows that VQ-RED generates much less rtt delay than DCF, especially when the buffer size is large. This is because the VQ-RED effectively controls the queue length which is achieved by controlling the length of the VQs before the packets are put into the real queue.

- Total goodput: the only disadvantage of VQRED, compared with DCF, is that it sacrifices a little bandwidth. This can be seen in Figure 6 .

\subsection{Effect on TCP Flows}

We set the sending rate of UDP flows to $2.0 \mathrm{Mbps} /(m+n)$, the buffer size to $100, n=8$, and do simulations with different value of $m(1,2,4,6, \ldots 16)$. The results are shown from Figure 12 to Figure 14.

Figure 12 shows the fairness among flows in the same direction. We can see that VQ-RED works very well with UDP and TCP traffic no matter what proportion of the uplink flow number and downlink flow number is. Although standard DCF also works well with UDP traffic, for TCP traffic, the bandwidth distribution is very unfair among the flows and this 
situation becomes worse as the number of flows increases.

Figure 13 shows the downlink/uplink fairness. We can see that under DCF, (1) the uplink TCP traffic totally overwhelms the downlink TCP traffic no matter what proportion of the uplink number of flows and downlink number of flows is; (2) for UDP traffic, the downlink/uplink unfairness is very obvious and the unfairness becomes worse as the uplink flow number increases. Under VQ-RED, the downlink/uplink fairness is greatly improved, we can see that the proportion of average goodput of downlink flows and uplink flows is higher than 0.8.

Figure 14 shows the achieved TCP goodput, UDP goodput and total goodput with VQ-RED and $\mathrm{DCF}$, respectively. Note that we set the sending rate of UDP traffic as $2.0 \mathrm{Mbps} /(m+n)$, where $m$ is the number of uplink flows and $n$ is the number of downlink flows. From Figure 14 we can see that the UDP flows can achieve this rate under VQ-RED while can not achieve under DCF. The only disadvantage of VQ-RED, compared with DCF is that its total achieved goodput (i.e. sum of TCP goodput and UDP goodput) is less than that of DCF.

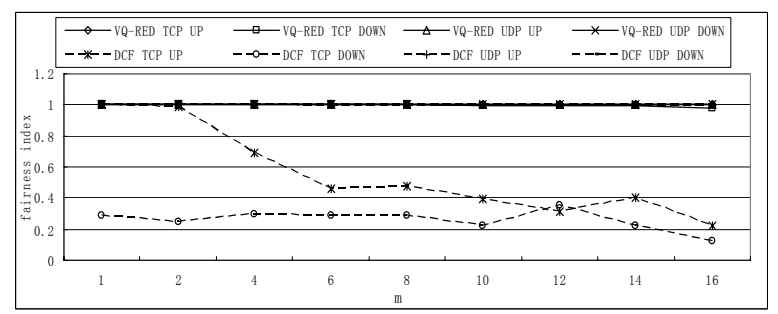

Figure 12 fairness among flows in the same direction (Buffer size $=100, n=8$, sending rate of UDP = $2 \mathrm{Mbps} /(\mathrm{m}+\mathrm{n}))$

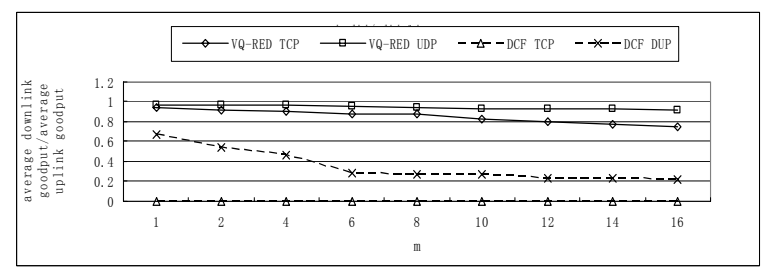

Figure 13 downlink/uplink fairness (Buffer size $=100, n=8$, sending rate of UDP $=$ 2Mbps/(m+n))

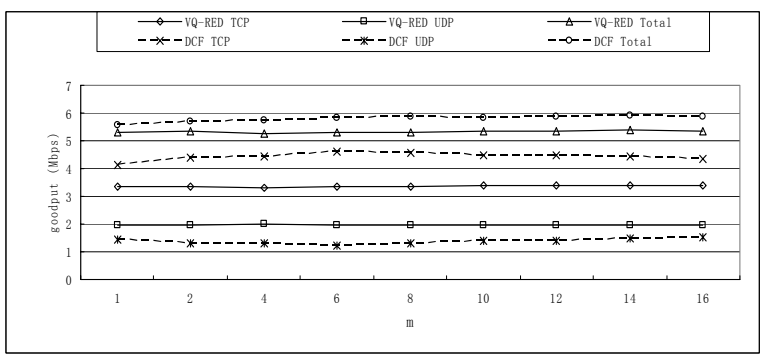

Figure 14 total achieved goodput (Buffer size = $100, n=8$, sending rate of UDP $=$ $2 \mathrm{Mbps} /(\mathrm{m}+\mathrm{n}))$

\subsection{Dynamic behavior of VQ-RED and DCF}

This simulation investigates the dynamic behavior of VQ-RED and DCF. This simulation lasts 400s. $m: n=5: 5$ in $[0,100] \mathrm{s}, \quad m: n=10: 5$ in $[100,200] \mathrm{s}$, $m: n=10: 10$ in $[200,300] \mathrm{s}$ and $m: n=5: 10$ in the $[300,400] \mathrm{s}$. The buffer size is set as 100 during the whole simulation and the sending rate of UDP traffic is $100 \mathrm{kbps}$. The results are shown in Figure 15 and Figure 16. We can see that VQ-RED works well under dynamic traffic.

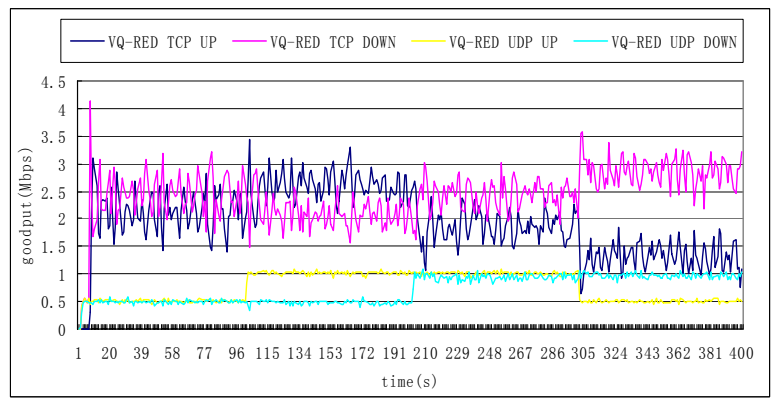

Figure 15 dynamic traffic under VQ-RED (Buffer size $=100$, sending rate of UDP traffic is $100 \mathrm{kbps}$ )

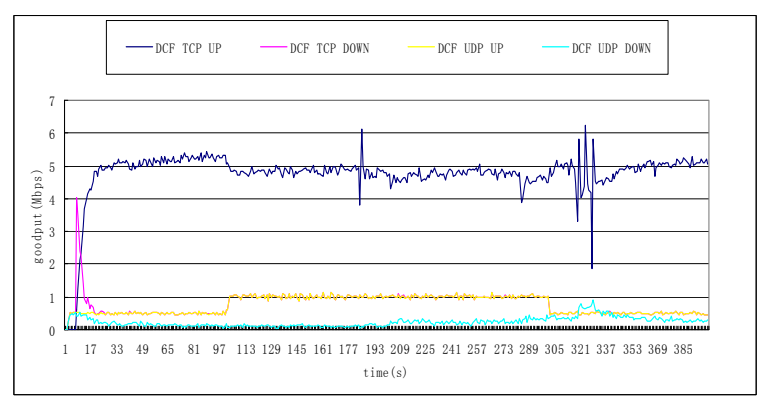

Figure 16 dynamic traffic under DCF (Buffer size $=100$, sending rate of UDP traffic is $100 \mathrm{kmps}$ ) 


\section{Conclusions}

In this paper, we proposed a software upgradebased approach, named VQ-RED to address the fairness problems (including downlink/uplink fairness and fairness among flows in the same direction) in the infrastructure 802.11 WLANs. Simulation results show that compared with standard DCF, VQ-RED not only greatly improves the fairness, but also generates much less delay, especially when the buffer size at AP is large. The only disadvantage of VQ-RED is that it sacrifices some bandwidth.

\section{References}

[1] Wireless LAN medium access control (MAC) and physical layer (PHY) specifications. Aug. 1999.

[2] S. Pilosof, R. Ramjee, D. Raz, Y. Shavitt and P. Sinha, "Understanding TCP fairness over wireless LAN", In Proc. IEEE INFOCOM'03, Apr. 2003.

[3] A. Detti, E. Graziosi, V. Minichiello, S. Salsano, and V. Sangregorio, "TCP Fairness Issues in IEEE802.11 Based Access Networks" submitted paper.

[4] M. Bottigliengo, C. Casetti, C.Chiasserini, M. Meo,"Smart Traffic Scheduling in 802.11 WLANs with Access Point", In Proc. IEEE VTC 2003, Oct. 2003.

[5] S. Yi, M. Kappes, S. Garg, X. Deng, G. Kesidis, and C. R. Das, "Proxy-RED: An AQM scheme for Wireless Local Area Networks," In Proc. IEEE ICCCN, 2004.

[6] S. W. Kim, B. S. Kim, Y. Fang, "Downlink and Uplink Resource Allocation in IEEE 802.11 Wireless LANs", In IEEE Trans. on Vehicular Technology, Vol. 54, No. 1, Jan. 2005, pp. 320-327.

[7] H. Y. Wei, C. C. Chiang, Y. D. Lin, "Co-DRR: An Integrated Uplink and Downlink Scheduler for Bandwidth Management over Wireless LANs", In IEICE Trans. on Communications, To Appear.

[8] UCB/LBNL/VINT Network Simulator - NS (version 2), http://www-mash.cs.berkeley.edu/ns/.

[9] R. Jain et. al., "A Quantitative Measure of Fairness and Discrimination For Resource Allocation in Shared Computer Systems", DEC RR TR-301, Sep. 1984. 